corresponding OS were 98.1 and 26.6 months, respectively $(\mathrm{HR}=0.34, P=0.003)$. For $\mathrm{MVI}(+)$ patients, the median PRS in RR/RFA group $(n=35)$ and TACE group $(n=137)$ were 15.9 and 10.7 months, respectively $(\mathrm{HR}=0.67, P=0.105)$. The corresponding OS were 23.5 and 16.8 months, respectively $(\mathrm{HR}=0.66, P=0.087)$. After matching, the dominance of RR/RFA over TACE remained in MVI(-) patients for both PRS (62.3 vs 18.5 months; $\mathrm{HR}=0.37,95 \% \mathrm{CI}=0.15-0.96$; $P=0.033)$ and $\mathrm{OS}(98.1$ vs 33.3 months; $\mathrm{HR}=0.31$, $P=0.008)$. No significant difference was found in $\mathrm{MVI}(+)$ patients for either PRS (15.9 vs 15.6 months; $H R=0.83$, $95 \% \mathrm{CI}=0.44-1.55 ; P=0.554)$ or OS (23.5 vs 28.1 months; $\mathrm{HR}=0.90, P=0.752)$. The cost of TACE group was significant lower than that of the RR/RFA group for both MVIpositive patients $(P=0.007)$ and MVI-negative patients $(P<0.001)$.

Conclusions For MVI-negative patients, RR/RFA provided better survival than TACE while for MVI-positive patients, TACE was recommended.

\section{IDDF2019-ABS-0086 CLINICAL PROFILE OF PATIENTS WITH HEPATOCELLULAR CARCINOMA AT ST. LUKE'S MEDICAL CENTER AND CARDINAL SANTOS MEDICAL CENTER FROM 2003- JULY 2018}

${ }^{1}$ John Alfredo Raphael Pangilinan*, ${ }^{2}$ Nicodemus Ong, 'Diana Alcantara-Payawal, ${ }^{2}$ lan Cua. ${ }^{1}$ Cardinal Santos Medical Center, Philippines; ${ }^{2}$ St. Luke's Medical Center, Philippines

\subsection{6/gutjnl-2019-IDDFabstracts.258}

Background In the Philippines, data is limited on the characteristics of patients with hepatocellular carcinoma (HCC). Recent studies from other neighboring Asian countries suggests a change in trend in the epidemiology of HCC. This study aims to update the data, describe the etiology and clinical profile of patients with HCC at 2 tertiary referral centers in the Philippines.

Methods This is a two-center retrospective, descriptive study of all adult patients with hepatocellular carcinoma at St. Luke's Medical Center, Quezon City (SLMC-QC) and Cardinal Santos Medical Center (CSMC). Clinical profile data, specifically patient's age, gender, presence or absence of liver cirrhosis, Child's Pugh score, and registered etiology of HCC (HBV, HCV, alcoholic liver disease, nonalcoholic fatty liver disease, others) was obtained and recorded in a Microsoft Excel. Data were analyzed using descriptive statistics.

Results A total of 1260 subjects were included in this study. Patients were predominantly male $(76.1 \%)$ with a mean age of 63 . The majority (83.3\%) developed HCC under a background of liver cirrhosis, with baseline liver function under Child-Pugh B at 51.8\%, followed by Child's A (23\%) and Child's C (17.6\%). Hepatitis B (44.3\%) is the most common etiology, followed by NAFLD (16\%). Hepatitis B was also the most predominant etiology for HCC in noncirrhotic HCC patients.

Conclusions Patients with HCC from both our centers are mostly males, with a mean age of 63. Majority developed HCC on a background of liver cirrhosis, with hepatitis B being the most common etiology followed by NAFLD.

\section{IDDF2019-ABS-0087 FIFTEEN YEARS OF HEPATOCELLULAR CARCINOMA, A PARADIGM SHIFT FROM INFECTIOUS TO NON-INFECTIOUS ETIOLOGY}

John Alfredo Raphael Pangilinan* . Cardinal Santos Medical Center, Philippines

\subsection{6/gutjnl-2019-IDDFabstracts.259}

Background To determine the etiologies and to describe the clinical profile of patients with hepatocellular carcinoma using the Child-Pugh Score at Cardinal Santos Medical Center in two-time frames (2003-2010, 2011-2018)

Methods This is a single-center retrospective, descriptive study of all adult patients with hepatocellular carcinoma at Cardinal Santos Medical Center (CSMC) in a fifteen-year span (2003-2018). Clinical data, including essential demographics were obtained. Chart review and database review was done.

Results Total study subjects from 2003-2018 were 674, with $74 \%$ of subjects being male and $26 \%$ female. Child's Pugh B was the most common, representing $67 \%$ of cases.

Based on etiology, Hepatitis B was predominant, totaling to $56 \%$ of cases, followed by NAFLD at $18 \%$ then ALD at $14.6 \%$. Even with the majority of subjects, specifically $56 \%$ representing HCC with $\mathrm{HBV}$ etiology, there was a significant drop in the number of cases in Time 2. There was also a significant increase in the cases of NAFLD, with a significant decrease in the cases of ALD.

Comparison of data showed a statistically significant change in the infectious and non-infectious causes of HCC. Time 1 showed a predominance of HCC with infectious etiology, representing $67 \%$ of all cases, with most cases from Hepatitis B. Time 2 showed a $22 \%$ decrease in this etiology, which could be inferred to be an effect of vaccination readily producing this endpoint. An interesting note is the prominent increase in the number of HCC cases arising from non-infectious causes, with a modest $21 \%$ of cases in time 1 , to a little over two times of that in time 2. (figure 1)

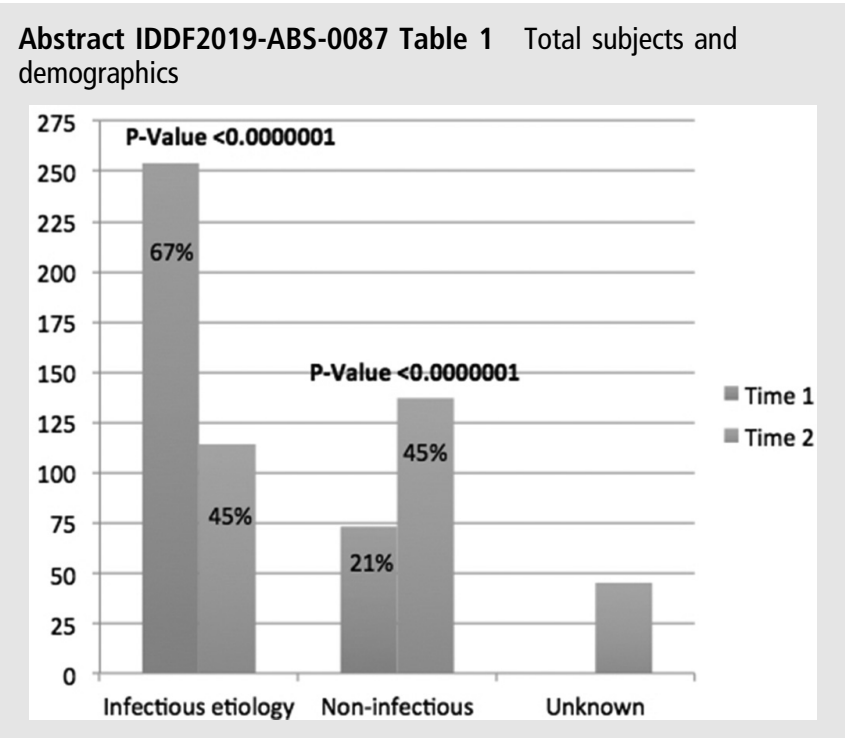

